\title{
Sustainable Polyimidazolium Networks as Versatile Hydrogel Materials
}

\author{
Steffen Tröger-Müller and Clemens Liedel*i) \\ Department of Colloid Chemistry, Max Planck Institute of Colloids and Interfaces, Research Campus Golm, 14476 Potsdam, \\ Germany
}

\section{Supporting Information}

ABSTRACT: This paper discusses the formation and properties of sustainable polyelectrolyte networks. The networks are formed from renewable precursors in a simple and green approach and feature a high density of positive charges in the main chain of the polymer backbone. Furthermore, zwitterionic systems with positive charges in the main chain and carboxylate side groups are presented. Depending on the cross-linking density and nature of the polyelectrolyte, flexible elastic or brittle networks are available which may form hydrogels upon absorption of up to $1500 \%$ water respective to their own mass. Reversible swelling in contact with different solvents furthermore envisions possible applications as smart material or selective membrane.

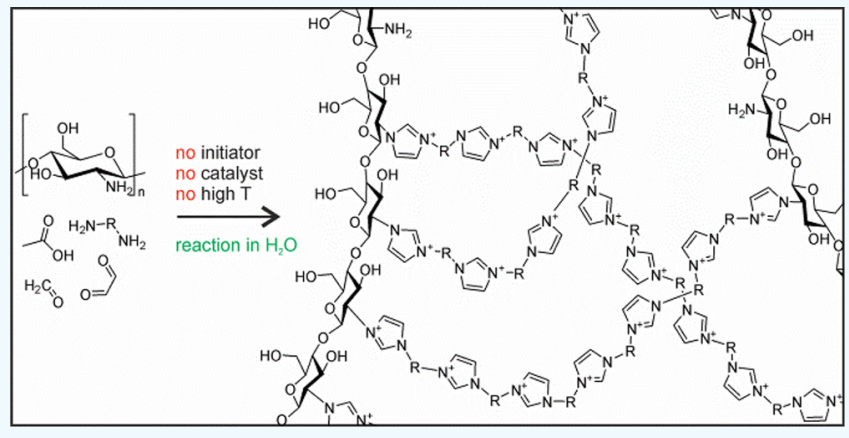

KEYWORDS: polyelectrolyte network, hydrogel, imidazolium, sustainable chemistry, sensor, smart membrane, chitosan

\section{INTRODUCTION}

Recent years have seen increasing awareness of environmental concerns and increasing visibility of adverse effects related to an unsustainable way of life. ${ }^{1-6}$ In order to tackle these challenges, such observations demand for new highly versatile and sustainable platform materials with adapted chemical synthesis routes. One class of chemical compounds which is promising in this regard is ionic liquids (ILs), because each ILtype, normally grouped by their cation-type, can be individually tailored to suit a variety of applications with only minimal variations of the general synthetic approach. ${ }^{7-12}$

Unfortunately, while ILs do meet the expectations of being highly tunable, rather sustainable, and offering unique characteristics, their liquid state hardly qualifies them as materials to be used in everyday life. In order to transfer their advantages to materials science, consequently multiple approaches like blending with polymers, ${ }^{13,14}$ gelation, ${ }^{15,16}$ or polymerization $^{17}$ of ILs have been applied with the latter defining a new class of polymers known as polymerized ionic liquids (PILs). ${ }^{18,19}$ PILs successfully combine the unique characteristics of ILs with materials science but, like many ILs, often suffer from rather extensive multistep synthesis requirements. Usually a neutral precursor molecule (e.g., imidazole) is synthesized from small molecules first, which is then transferred to the desired ionic species by addition of substituent(s) at a heteroatom (e.g., formation of imidazolium). In the case of a PIL, one or more of these substituents contain a polymerizable group or can be transformed to a polymerizable group, which in a next step is used to fixate ionic liquid functionalities in a polymer architecture. This route to
IL and PIL synthesis hence often includes multiple alkylation steps which contribute to an undesirable ecological and economical footprint. ${ }^{20}$

Even though the above-mentioned approach is the standard method in many laboratories working in this area of research, alternatives exist. Especially the imidazolium cation, one of the most intensely studied IL-cations, has the benefit of being available via a very efficient route that directly forms the quaternized cation from small molecules such as formaldehyde, glyoxal, and amines in the presence of a weak organic acid like acetic acid. This so-called modified Debus-Radziszewski reaction $(\mathrm{mDRr})$ has been utilized by several research groups in the past years. ${ }^{21,22}$ As we have recently pointed out, this reaction works quantitatively, without the use of auxiliaries, catalysts, or high temperatures. All reagents and a wide array of chemicals with similar functions can be obtained from renewable resources, demonstrating the sustainability and versatility of the approach. ${ }^{23}$ As a further testament to this versatility, the use of the $\mathrm{mDRr}$ in polymer science has recently received increasing attention:

Polymers with pendant amine functionalities may be crosslinked in this way. ${ }^{24,25}$ Similarly, linear polyelectrolytes ${ }^{26-28}$ or covalent organic frameworks (COFs) ${ }^{29}$ may be synthesized via a polycondensation-like approach by using diamines or molecules with multiple amine functionalities, respectively. Figure 1 schematically illustrates this modified Debus-

Received: May 28, 2019

Accepted: August 23, 2019

Published: August 23, 2019 


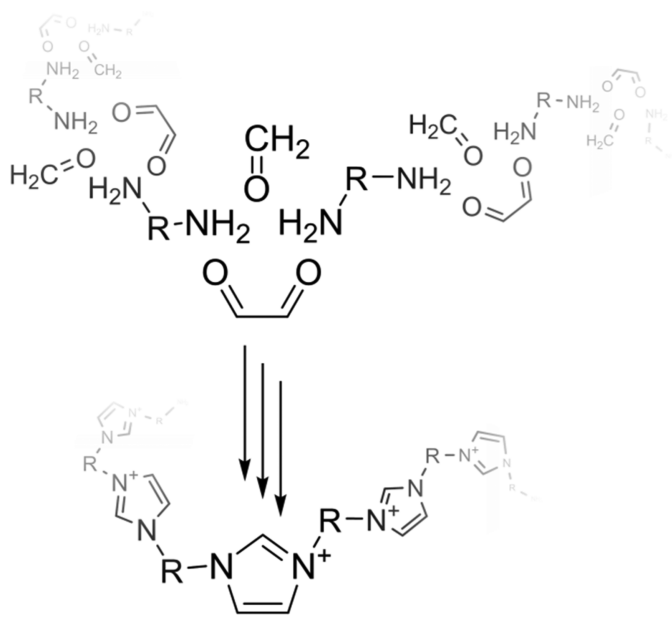

Figure 1. Schematic expression of the synthesis of linear main-chain polyelectrolytes via the modified Debus-Radziszewski polymerization reaction. Counterions are omitted for clarity of presentation.

Radziszewski polymerization reaction. Next to the advantages in terms of economy and sustainability mentioned above, the approach is appealing because of the unusual architecture of the poly(imidazolium) chains with charged sites in the polymers' main chains. Also, although the reaction releases water and is formally a polycondensation reaction, it is not dependent on exact stoichiometry and as such can be driven forward by applying stress to the chemical equilibrium. ${ }^{28}$

Sirviö et al. ${ }^{24}$ previously showed that the $\mathrm{mDRr}$ also cross links primary amine groups in the biopolymer chitosan upon formation of imidazolium groups. This cheap polymer carries an amino group in almost every repeating unit and is available from chitinous waste-biomass such as shrimp shells by deacetylation. As such, it contributes significantly to the sustainability of the formed network. The main obstacles of using chitosan in synthesis, however, are on the one hand its large molecular weight and thus bad solubility, which can be overcome by using an aqueous acetic acid solution as a solvent, and on the other hand the tightness of networks due to the insufficient flexibility of linkers between chitosan chains. In contrast, linear poly(imidazolium) polyelectrolytes as presented by Lindner and Yuan et al. $^{26-28}$ are flexible but not usable for new materials due to solubility in common solvents. Consequently, advantages of both approaches need to be combined in order to form new platform materials based on poly(imidazolium) polymers.

In general, upon introduction of monomers with multiple amine functionalities in a low amount together with monomers for linear polymer formation, polymer networks may be formed. The aim of this study is to use this concept and combine the synthesis of a polymer and a COF via the modified Debus-Radziszewski polymerization approach to produce a platform for tunable and sustainable materials, for example, to be used as sensors or charge sensitive membranes. In this regard, we combine the use of linear diamines like cadaverine, 4,7,10-trioxa-1,13-tridecanediamine, or lysine with the use of chitosan as a sustainable molecule that carries more than two amino groups to form rather flexible networks based on the mDRr. In contrast to conventional polymerized ionic liquids, these networks are obtained in a one-pot reaction from the small molecular starting material, and we will in the following refer to them as polyelectrolyte networks (PENs).

\section{RESULTS AND DISCUSSION}

PENs X-Y are primarily based on cadaverine, 4,7,10-trioxa1,13-tridecanediamine (in the following also denoted oligoether), or lysine and denoted C-Y, T-Y, and L-Y, respectively $(\mathrm{X}=\mathrm{C}, \mathrm{T}$, or $\mathrm{L})$. For formation of networks, however, a low proportion of 3,5 , or $7 \%$ of the amines in the reagent mixture were also chitosan-based, resulting in PENs X-3, X-5, and X-7, respectively $(Y=3,5$, or 7$)$. It should be noted that chitosan is not completely deacetylated, which we accounted for accordingly (for details of the synthesis we refer to the Experimental Part). Figure 2 summarizes the different amines used in this study, a schematic of the formed polyelectrolyte network, and representative photos of the different PENs.

In order to classify the formed material, we estimate the length of the polymer chains. Although approximately 70-80 repeating units constitute comparable linear polyelectrolytes, ${ }^{28}$ in our case between $3 \%$ and $7 \%$ of amine functionalities (every 14 th to $33 \mathrm{rd}$ amine) are chitosan-based, which statistically results in average poly(imidazolium) chain lengths between chitosan chains of 13-32 repeating units (approximately 2.3 to $10 \mathrm{kDa}$, depending on the type of diamine). Nonstoichiometry of the reagents may further decrease the chain length in the polycondensation-like reaction, even though the influence might be not as big as in conventional polycondensation reactions. ${ }^{28}$ Chitosan in the reagent mixture statistically terminates the polyelectrolyte chains at one or both ends, leading to a cross-linked network (Figure 2a). As chitosan with molecular weight of about $50-190 \mathrm{kDa}$ (median, $120 \mathrm{kDa}$ ) was used, this number largely exceeds the molecular weight of the individual poly(imidazolium) chains, so the resulting network might rather be described as chitosan backbone with grafted polyelectrolyte chains or different chitosan chains cross-linked by poly(imidazolium) polyelectrolyte chains. Because of poly(imidazolium) chains in the range of up to $10 \mathrm{kDa}$, still rather open networks are obtained.

IR-data confirming the structure of the PENs is discussed in the Supporting Information (Figures S1-S10). The resulting cadaverine- and oligoether-based networks are rubber-like and rather supple. PENs based on lysine in contrast are brittle at room temperature. Figure $2 \mathrm{~b}$ shows photos of representative examples. In order to investigate this characteristic in more detail, we next discuss the thermal behavior. Table 1, Figure S11, and Figure S12a give an overview over thermal decomposition and glass transition temperatures of the synthesized PENs.

All PENs show more or less continuous mass loss from the beginning of the thermogravimetric analysis (TGA) and a major decomposition step around $275-300{ }^{\circ} \mathrm{C}$. The amount of chitosan in the PENs has no significant effect on thermal behavior. A substantial difference is however observed when comparing lysine based PENs to the other samples. The former generally exhibit lower initial mass loss before thermal decomposition, an increased decomposition temperature, and more remaining mass after thermal decomposition. We attribute this behavior to a significant difference in architecture: while PENs based on cadaverine or 4,7,10trioxa-1,13-tridecanediamine are cationic polyelectrolyte networks (c-PENs) with acetate counterions, lysine-based PENs are of zwitterionic nature, because $\mathrm{p} K_{\mathrm{A}}$ (lysine) $<\mathrm{p} K_{\mathrm{A}}$ (acetic acid) (z-PENs) with main chain imidazolium and carboxylate functionalities. The different charge likely results in stronger 


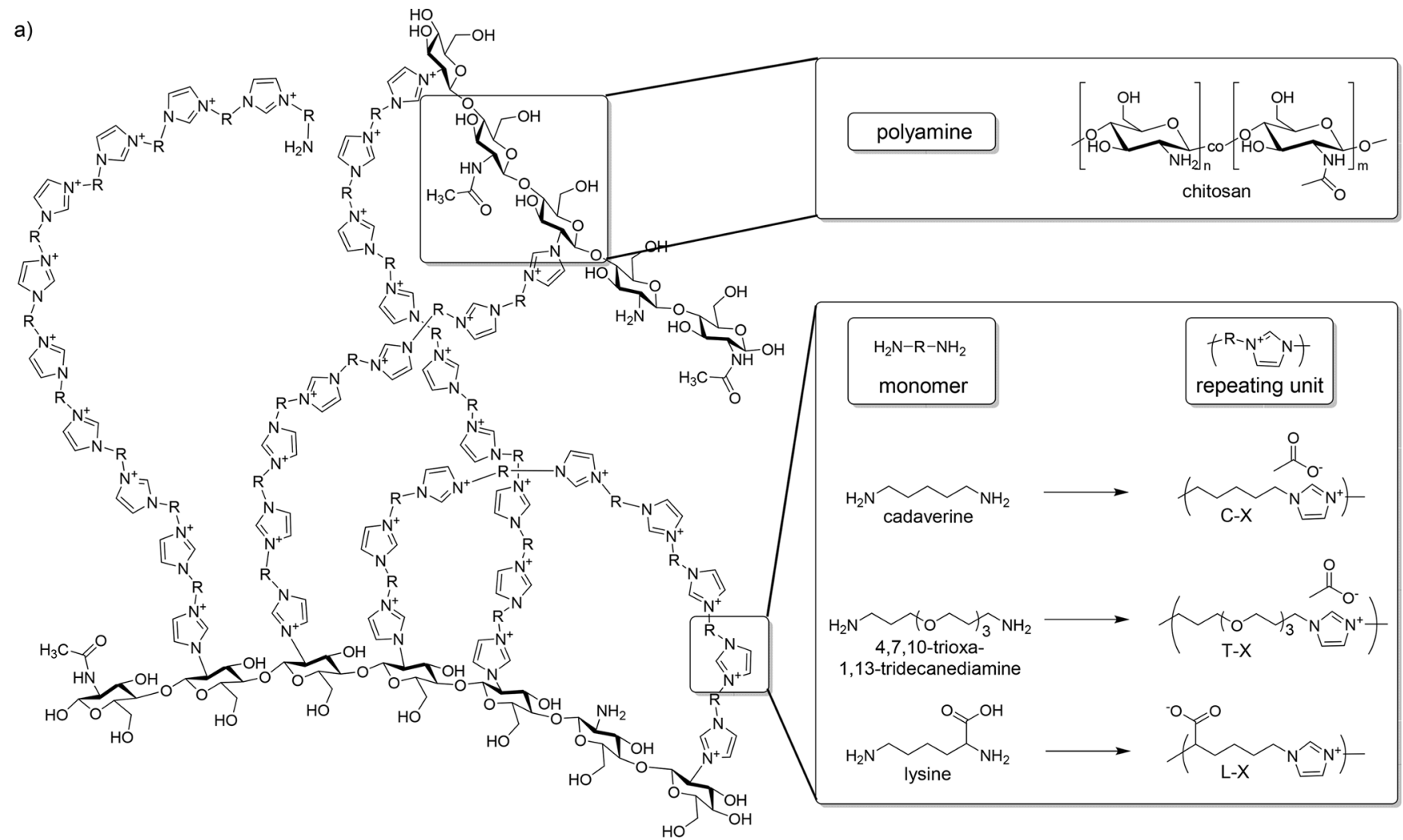

b)

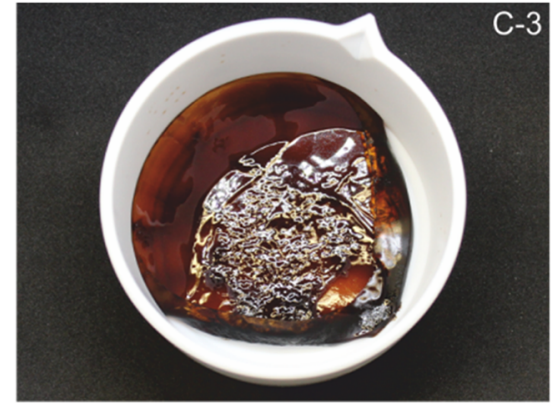

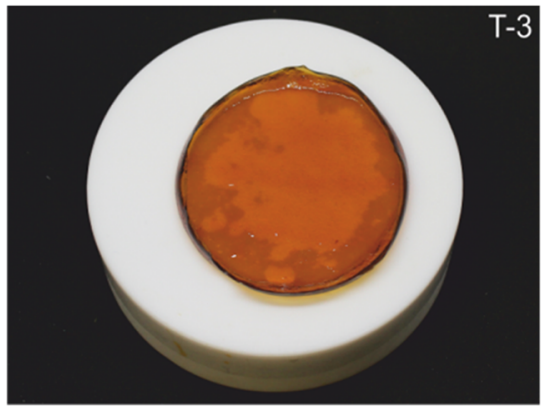

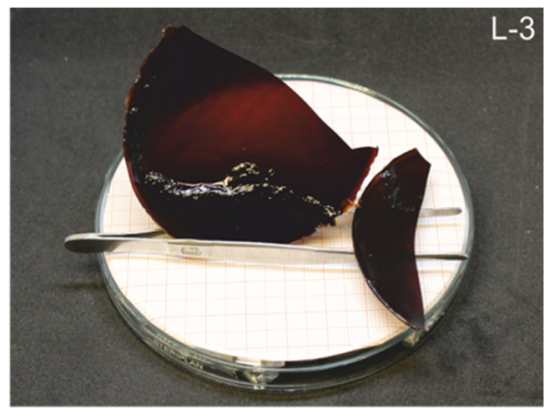

Figure 2. (a) Schematic structure of the polyelectrolyte networks. Any tacticity is for convenience of display only. Counterions are omitted in the schematic drawing for image clarity. The monomers and repeating units of PENs are shown in the drawn out rectangles. Different amounts of chitosan were used as sustainable polymeric species with multiple amine functionalities to cross-link linear polyelectrolyte chains. Indices $n$ and $m$ in the structure of chitosan indicate that not all but only approximately $80 \%$ of glucosamine monomers are deacetylated $(n \approx 80 \%)$. (b) Photos of PENs C-3, T-3, and L-3 after synthesis. Photos were taken by the authors. Names of the PENs present the first letter of the used monomer followed by the percentage of chitosan-based amino groups during synthesis.

Table 1. Overview over the Thermal Behavior of Synthesized PENs with Heating/Cooling Rates of $10 \mathrm{~K} \mathrm{~min}{ }^{-1} \cdot{ }^{a}$

\begin{tabular}{|c|c|c|c|c|c|}
\hline diamine & sample & mass loss before decomposition /\% & $T_{\mathrm{D}} /{ }^{\circ} \mathrm{C}$ (mass loss $\left./ \%\right)$ & mass loss after decomposition/\% & $T_{\mathrm{G}} /{ }^{\circ} \mathrm{C}$ \\
\hline \multirow[t]{3}{*}{ cadaverine } & C-3 & 29.9 & $280.4(61.7)$ & 3.7 & -31.4 \\
\hline & C-5 & 31.0 & $281.9(59.2)$ & 4.4 & -22.5 \\
\hline & $\mathrm{C}-7$ & 30.5 & $282.9(57.6)$ & 5.6 & -28.9 \\
\hline \multirow[t]{3}{*}{ 4,7,10-trioxa-1,13-tridecane-diamine } & $\mathrm{T}-3$ & 27.9 & $275.2(67.1)$ & 2.7 & -42.0 \\
\hline & $\mathrm{T}-5$ & 22.8 & $276.2(69.1)$ & 4.3 & -47.6 \\
\hline & $\mathrm{T}-7$ & 23.3 & $279.7(67.8)$ & 4.3 & -34.4 \\
\hline \multirow[t]{3}{*}{ lysine } & L-3 & 10.7 & $293.4(69.3)$ & 11.6 & 84.0 \\
\hline & L-5 & 12.3 & $302.9(68.9)$ & 9.7 & 99.8 \\
\hline & L-7 & 9.4 & $295.4(73.4)$ & 10.1 & 76.4 \\
\hline
\end{tabular}

${ }^{a}$ Decomposition temperatures $T_{\mathrm{D}}$ describe the absolute minimum of the first derivative of the TGA curves in the range up to $600{ }^{\circ} \mathrm{C}$. The corresponding mass loss represents mass loss in the range $225-400{ }^{\circ} \mathrm{C}$. The difference to $100 \%$ corresponds to the remaining mass at $600{ }^{\circ} \mathrm{C}$. Glass transition temperatures $T_{\mathrm{G}}$ represent the inflection points of DSC curves (third heating). 
interactions between the chains, which leads to higher decomposition temperatures. The presence of additional ionic interchain interactions between carboxylate and imidazolium functionalities is also reflected in significantly higher glass transition temperatures of the z-PENs in comparison to the c-PENs.

It is worth noting that samples presented here were treated at $60{ }^{\circ} \mathrm{C}$ overnight during synthesis (see Experimental Part) and stored at ambient conditions before analysis in order to demonstrate the behavior in possible actual applications. The significant reduction of mass prior to the major decomposition step may indicate low molecular weight components (lmc) like water incorporated in the material that evaporate at lower temperatures compared to the polymer's degradation temperature. Indeed, exposure of T-5 samples to elevated temperature for prolonged time $\left(24 \mathrm{~h}, 100{ }^{\circ} \mathrm{C}\right)$ results in a weight loss of $21.2 \%$, which is comparable to the results in Table 1 (mass loss before decomposition). In TGA investigations, right after such a thermal treatment step (Figure $\mathrm{S} 12 \mathrm{~b}$ in the Supporting Information), only $2.6 \%$ of the mass is lost before decomposition. The decomposition temperature is unaffected. Interestingly, exposure of the sample to ambient air after thermal treatment at $100{ }^{\circ} \mathrm{C}$ for $24 \mathrm{~h}$ results in saturation with humidity again (recovery to $96.3 \%$ of the initial mass within 24 h). Subsequent TGA investigations again show mass loss before thermal decomposition of $19.4 \%$ (Figure S12b in the Supporting Information).

In order to investigate the composition of PENs and the nature of $1 \mathrm{mc}$ in more detail, we conducted thermogravimetric analysis coupled to mass spectrometry (TGA-MS) on sample T-5 (see Figure S13 in the Supporting Information). For brevity only selected, representative curves are displayed. Three sets of curves are investigated in detail: $\mathrm{m} / z 17$ and 18 are interpreted to show hydroxyl and water radicals, respectively, which indicates the evaporation of water during heating the PEN. The detection of $\mathrm{m} / z 43$ and 60 indicates acetic acid, and $m / z 12$ and 44 are characteristic for the evolution of $\mathrm{CO}_{2}$ and part of the mass spectrum of acetic acid (if a hard ionization method is used that fragments the molecule, such as electron ionization). The measurement reveals three regions of interest, where low molecular weight components are detected: before (region 1), during (region 2), and after the major decomposition event (region 3). In region 1 , water and acetic acid are observed. We attribute this observation to evaporation of residual water and acetic acid from the intact PEN, confirming the hygroscopic behavior and difficulty to remove these components, which are available in excess during synthesis, completely. Region 2 corresponds to the decomposition of the network. Consequently decomposition products are detected. When $\mathrm{C}-\mathrm{N}$ bond dissociation results in neutralization of the positive charges in the PENs, the electrostatically bound acetate is released. As a result of this loss as well as the loss of charges within the PEN, the hydrogen bonding capacity is reduced, and consequently bound water is released from the PEN in region 2, too. The release of water and acetic acid is also indicated in region 3 . We assume that the process is analogue to region 2 . The significant difference in temperature may relate to structural differences of the imidazolium moieties within the PEN. As the mass loss in region 2 is much larger than in region 3, we assume that region 2 relates to decomposition of the poly(imidazolium) chains connecting the chitosan chains. Region 3 may relate to $\mathrm{C}-\mathrm{N}$ bond dissociation of chitosan-bound imidazolium rings.
TGA-MS measurements confirm the presence of $1 \mathrm{mc}$ within the PENs. Such additives to polymers generally decrease the apparent $T_{\mathrm{G}}$. Still, the observed trend of lower $T_{\mathrm{G}}$ in 4,7,10trioxa-1,13-tridecanediamine based PENs compared to cadaverine based PENs does not correlate with the amount of $1 \mathrm{mc}$, as the initial mass loss is highest in the case of cadaverine-based PENs. Consequently, although lmc surely contribute to the observed glass transition temperatures, rubberlike behavior at room temperature is unlikely to be caused by low molecular constituents alone. The trend in $T_{\mathrm{G}}$ instead likely is a combined result of chain flexibility, intermolecular interactions, and the presence of low molecular constituents. The z-PENs feature lower lmc content, comparatively short chains between imidazolium cations, and the presence of covalently bound anions in addition to the imidazolium cations, resulting in stronger interactions between the chains. Consequently, the highest glass transition temperatures are presented in the $\mathrm{z}$ PENs based on lysine.

As mechanical characteristics are a decisive factor in application, the synthesized PENs based on cadaverine and the oligoether were subjected to oscillatory stress-strain measurements to determine their mechanical behavior. Unfortunately, PENs based on lysine were too brittle to be investigated at room temperature (see Figure 2c), most likely as a result of the peculiarity discussed above. Figure 3 summarizes the results.

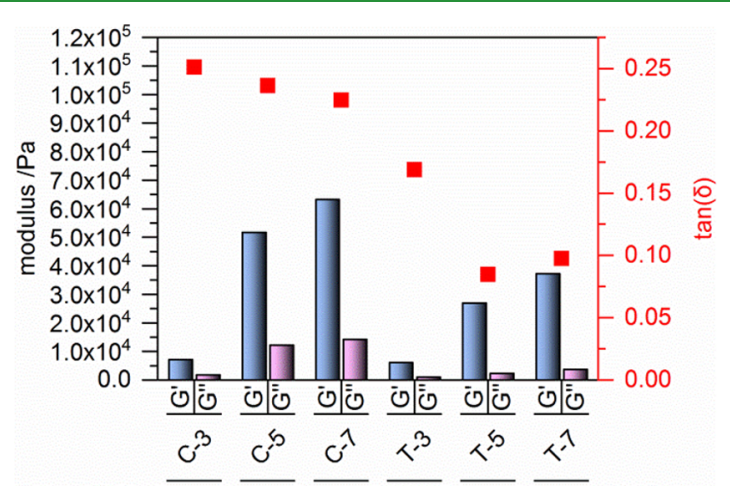

Figure 3. Storage modulus $\mathrm{G}^{\prime}$, loss modulus $\mathrm{G}^{\prime \prime}$ (bars), and loss factor $\tan (\delta)$ (red) of PENs based on cadaverine (C) and 4,7,10trioxa-1,13-tridecanediamine $(\mathrm{T})$ with 3,5 , or $7 \%$ of amine groups exchanged by chitosan-based amino groups during synthesis..

Comparing the storage modulus $G^{\prime}$ (representing elastic behavior) among the respective samples, higher values are observable in samples with higher amounts of chitosan. A significant increase of the storage modulus can be observed upon increasing the amount of chitosan cross-linker from C-3 to $\mathrm{C}-5$ and T-3 to T-5. This behavior may be explained by the rather high molecular weight of chitosan. As (i) the amount of chitosan in the reaction was calculated based on the amount of amine groups, (ii) each molecule of chitosan contains approximately 236-896 amine groups, and (iii) only $3 \%$ of amine groups in the reagent mixture for C-3 and T-3 were based on chitosan, each molecule of chitosan was on average mixed with 3900-15000 molecules of cadaverine or 4,7,10trioxa-1,13-tridecanediamine. This low concentration of chitosan chains in the final network may prevent sufficient entanglement and interconnection of the chitosan chains. Poly(imidazolium) chains in such samples may rather connect different amine groups of the same chitosan molecule 
(schematically shown as a "loop" in Figure 2a), and only at higher concentrations (C-5, C-7, T-5, T-7), entanglements and interconnections lead to the drastic increase of the storage modulus.

Generally, higher values of $G^{\prime}$ are obtained for the cadaverine-based systems, which is likely a result of the comparatively short length of the cadaverine molecule (five atoms between the amine groups) in contrast to the longer 4,7,10-trioxa-1,13-tridecanediamine molecule (13 atoms between the amine groups). A shorter chain as well as the consequently closer proximity of the positive charges on it result in lower flexibility and as such impose a stronger force to return into the original shape after the end of the application of force. The cadaverine-based PENs also feature a higher loss modulus ( $G^{\prime \prime}$, corresponding to viscous behavior) than the oligoether-based PENs. A higher amount of residual water and acetic acid in the cadaverine-based PENs (cf. Table 1, up to $30 \%$ residual $\mathrm{lmc}$ ), which effects a larger portion of force to dissipate irreversibly, may explain this observation. Comparing the moduli within each sample reveals that the storage modulus is generally larger than the loss modulus for all tested materials, independent of the frequency (cf. Figure S14 in the Supporting Information). Amplitude sweeps also show that $G^{\prime}>G^{\prime \prime}$ (cf. Figure S15 in the Supporting Information) which is also reflected in the loss factor $\tan (\delta)$ calculated from the obtained moduli of the samples (see Supporting Information), as shown in Figure 3 (right axis of ordinates). $\tan (\delta)$ is generally below 0.25 for all materials which indicates viscoelastic behavior with a dominant elastic part. ${ }^{6}$ The shape of the curves is typical for cross-linked polymers. ${ }^{6}$

In order to investigate the stability and adsorption properties, we next compare the behavior upon immersion in aqueous environment (pure $\mathrm{H}_{2} \mathrm{O}$ and $5 \% \mathrm{NaCl}(\mathrm{aq})$ ). Figure 4 illustrates swelling of the PENs in terms of increase of their mass.

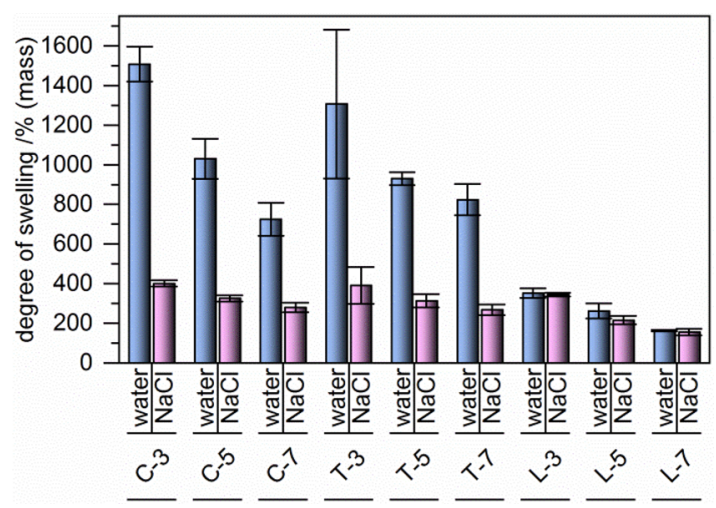

Figure 4. Degree of swelling in pure water or in $5 \% \mathrm{NaCl}(\mathrm{aq})$ in terms of mass change of different PENs as indicated.

c-PENs based on cadaverin or the oligoether with the same concentration of chitosan cross-linker swell to a comparable degree. In the case of all c-PENs, swelling in deionized water is generally about three times stronger than in sodium chloride solution, which is a result of the increased ionic strength of the salt solution in comparison to pure water influencing the osmotic pressure and thus reducing the swelling. The lysinebased systems behave differently and swell by approximately the same degree in both media. They also show the weakest swelling percentage of all tested networks, which is probably caused by their zwitterionic nature and the added electrostatic interactions.

The ability to accommodate water generally decreases with increased percentage of chitosan. As increasing chitosan content results in stronger cross-linking as discussed above, this observation is likely caused by the increasing rigidity of the networks (cf. Figure 3). Over all, the kind of charges in the polymer chains (only cations or cations and anions), followed by the amount of cross-linker, seems to be most important for the swelling behavior of the tested poly(imidazolium) networks.

Interestingly, the samples are insoluble in any solvent investigated. Instead, the PENs also swell in several organic solvents. Figure 5 summarizes the degree of swelling for several

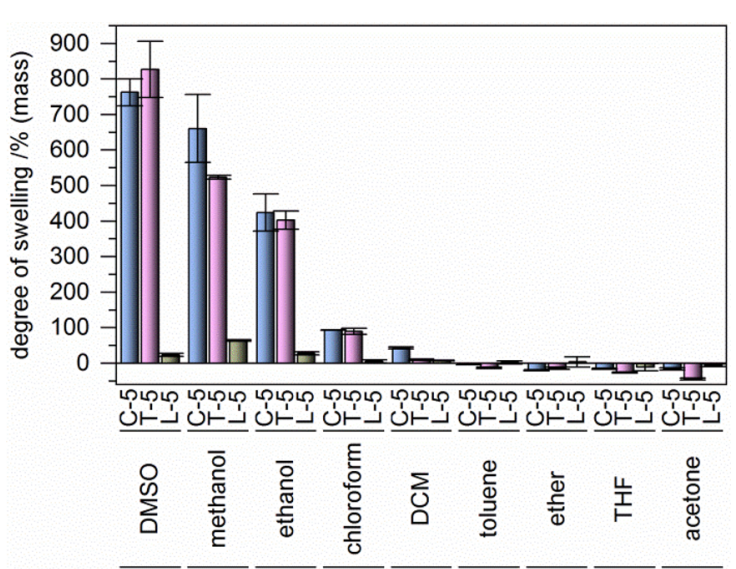

Figure 5. Degree of swelling in dimethyl sulfoxide (DMSO), methanol, ethanol, chloroform, dichloromethane (DCM), toluene, diethyl ether (ether), tetrahydrofuran (THF), or acetone in terms of mass change of different PENs as indicated.

common organic solvents. For better comparison, networks with the same amount of chitosan cross-linker are summarized to explore the influence of diamine on the swelling behavior.

Generally, swelling is less pronounced in organic solvents compared to aqueous media. Out of all studied organic solvents, solvent uptake is strongest upon immersion of the PENs in a large excess of dimethyl sulfoxide (DMSO) and follows the order DMSO > methanol > ethanol > chloroform > dichloromethane. For toluene, diethyl ether, tetrahydrofuran, and acetone, negative swelling of the PENs, that is, up to $45 \%$ mass loss upon immersion, is observed. We note that the structural integrity of the PENs is maintained also upon immersion in all tested organic solvents, indicating that the cross-linked material cannot be dissolved. Instead, residual water and acetic acid are likely being washed out of the polyelectrolyte network. Additional, short poly(imidazolium) chains that are not cross-linked into the network must be soluble here, as for some compositions and solvents, the mass loss is more intense than the possible percentage of water and acetic acid (cf. Table 1, mass loss before thermal decomposition).

Comparing the positive swelling behavior, it is similar for cadaverine and 4,7,10-trioxa-1,13-tridecanediamine based PENs. Yet again the behavior of lysine-based z-PENs is different as they are hardly affected by the solvents at all. Their zwitterionic state with hydrogen bonding sites in the form of carboxylates (i.e., hydrogen bond acceptors) bound to the polymer chains may be responsible for this observation as the 

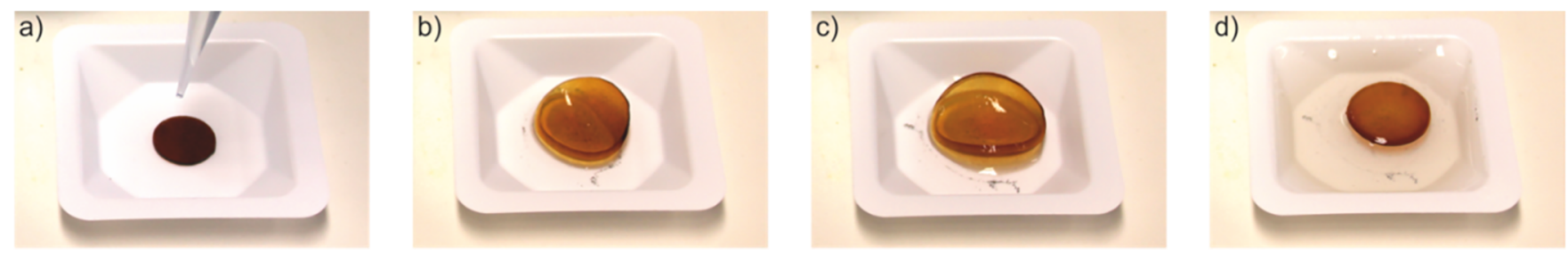

Figure 6. Photographs of swelling behavior in water and acetone. (a) The dry sample T-5 (91.1 mg) is wetted using deionized water. (b) After 45 min, the sample is significantly swollen and has taken up all water $(500 \mu \mathrm{L})$. (c) Further addition of water $(500 \mu \mathrm{L})$ and waiting $(1 \mathrm{~h})$ increases the amount of swelling. (d) If the same sample is then subjected to the presence of acetone $(5.0 \mathrm{~mL})$, the swelling is reversed substantially. Photos were taken by the authors.

investigated organic solvents are all aprotic. Generally, the swelling behavior of the three PENs in different solvents does not follow any immediately obvious trend or established polarity scale of solvents, even though uptake of polar solvents seems to be higher than nonpolar ones (compare DMSO, methanol, and ethanol). The incompatibility of the c-PENs with some solvents also does not follow any obvious trend. Instead, the amount of weight loss in acetone, THF, diethyl ether, and toluene is most likely caused by a combination of water and acetic acid being initially present in the PENs and the ability of the used solvent to mix with or dissolve water, acetic acid, and parts of the PEN which are not cross-linked. Further discussion of the swelling behavior with respect to the polarity of the solvent is included in the Supporting Information.

In order to emphasize the different swelling behavior even more, we also tested structural changes upon treatment with water and acetone as the two solvents which lead to the most intense swelling and shrinking, respectively.

In this regard, Figure $6 \mathrm{a}-\mathrm{c}$ illustrates the intense swelling of an oligoether based c-PEN with $5 \%$ of amine functionalities being chitosan-based (T-5) in water. Photographs of other PENs are included in the Supporting Information. In contrast to the above-described experiments, no excess of water was used. Instead, the 11-fold mass of water was added to the PEN in two equal portions. Figure $6 \mathrm{~b}, \mathrm{c}$ shows the resulting swollen PEN after the uptake of the added water (after $45 \mathrm{~min}$ and additional $60 \mathrm{~min}$, respectively). The water is absorbed almost completely as expected (cf. Figure 4). Upon addition of acetone to the swollen PEN (43-fold mass compared to the initial, not-swollen mass), the PEN shrinks significantly. Figure $6 \mathrm{~d}$ shows the resulting structure $10 \mathrm{~min}$ after addition of acetone. Shrinkage of the PEN confirms that immersion of the PENs in acetone results in a release of absorbed water. Slight discoloration of the solvent also indicates that indeed acetone may wash out small amounts of poly(imidazolium) chains of the material, without compromising the structural integrity though.

\section{CONCLUSION}

In conclusion, sustainable synthesis using various diamines yielded promising platform materials with tunable swelling behavior and mechanical properties. Flexible or brittle, cationic or zwitterionic, thermally stable networks were accessible in a benign and environmentally friendly reaction. While the individual cationic networks showed viscoelastic behavior with a dominant elastic part, zwitterionic networks were brittle with high glass transition temperatures. All PENs were stable in water and several organic solvents and were not soluble in any solvent under investigation. They swelled to a large degree in most polar solvents like water or DMSO and lost weight that was mainly caused by flushing low molecular weight components, like water, out of the network upon immersion in some organic solvents like diethyl ether, THF, or acetone. In cationic polyelectrolyte networks, careful selection of solvents enabled reversible swelling of the material.

The degree of swelling is easily tunable by choice of the chain connecting the imidazolium moieties as well as the amount of cross-linker used. Because of the easily adjustable synthesis conditions, besides the variation of diamines also the counterions of the polyelectrolytes may easily be tuned in future experiments. Solvent-selective swelling and high charge density may enable applications as sensor or charge sensitive membrane; also programmable materials are possible applications.

\section{EXPERIMENTAL PART}

Synthesis of PENs. In a typical synthesis, chitosan ("low molecular weight", Aldrich, degree of deacetylation approximately $80 \%)$ and the diamine were dissolved in a mixture of deionized water and acetic acid (analytical reagent grade, Fisher Scientific) and stirred for an hour at room temperature. A mixture of glyoxal (40\% solution in water, Roth) and formaldehyde $(37-38 \% \mathrm{w} / \mathrm{w}$, stabilized with methanol, Applicem Panreac) was added, and the solution was stirred for $10 \mathrm{~min}$. Afterward, the solution was cast into a PTFE Petri dish and dried in an air oven overnight at $75^{\circ} \mathrm{C}$. Details of the amounts of chemicals used for all samples discussed in this manuscript are provided in the Supporting Information.

Analysis. Details of the analysis by IR, DSC, TGA, TGA-MS, rheology, and investigations of the swelling behavior in various solvents are described in detail in the Supporting Information.

Safety. All PENs presented in this study are new compounds with yet uninvestigated properties regarding health and safety. They consequently must be treated with caution.

\section{ASSOCIATED CONTENT}

\section{S Supporting Information}

The Supporting Information is available free of charge on the ACS Publications website at DOI: 10.1021/acsapm.9b00491.

Experimental details, IR, DSC, TGA, TGA-MS, Rheology graphs, additional swelling experiments (PDF)

\section{AUTHOR INFORMATION}

\section{Corresponding Author}

*E-mail: Clemens.Liedel@mpikg.mpg.de.

ORCID

Clemens Liedel: 0000-0002-2323-9431

Notes

The authors declare no competing financial interest. 


\section{ACKNOWLEDGMENTS}

We highly appreciate help in the laboratory by Jessica Brandt. We further thank Antje Völkel for thermal analysis measurements, Albert Sanz de León for help with rheology measurements, and Markus Antonietti and Ivan Ilic for fruitful discussions. Finally, we acknowledge financial support by the Max Planck Society and the Max Planck Institute of Colloids and Interfaces. Open Access fees were covered by the Max Planck Society.

\section{REFERENCES}

(1) Crutzen, P. J. Geology of mankind. Nature 2002, 415, 23.

(2) Steffen, W.; Crutzen, P. J.; McNeill, J. R. The Anthropocene. Ambio 2007, 36, 614-621.

(3) NASA. GLOBAL CLIMATE CHANGE: Vital Signs of the Planet. The consequences of climate change. https://climate.nasa. gov/effects/ (accessed February 7, 2018)

(4) Caesar, L.; Rahmstorf, S.; Robinson, A.; Feulner, G.; Saba, V. Observed fingerprint of a weakening Atlantic Ocean overturning circulation. Nature 2018, 556, 191-196.

(5) Zhao, S.; Lü, B.; Li, R.; Zhu, A.; Wu, C. A preliminary analysis of fishery resource exhaustion in the context of biodiversity decline. Sci. China: Earth Sci. 2016, 59, 223-235.

(6) Arthington, A. H.; Dulvy, N. K.; Gladstone, W.; Winfield, I. J. Fish conservation in freshwater and marine realms. Aquatic Conserv: Mar. Freshw. Ecosyst. 2016, 26, 838-857.

(7) Mallakpour, S.; Dinari, M. Ionic Liquids as Green Solvents: Progress and Prospects. In Green Solvents II; Mohammad, A., Inamuddin, D., Eds.; Chemistry; Springer Netherlands: Dordrecht, 2012; pp 1-32.

(8) Plechkova, N. V.; Seddon, K. R. Applications of ionic liquids in the chemical industry. Chem. Soc. Rev. 2008, 37, 123-150.

(9) Badgujar, K. C.; Bhanage, B. M. Factors governing dissolution process of lignocellulosic biomass in ionic liquid: current status, overview and challenges. Bioresour. Technol. 2015, 178, 2-18.

(10) Tang, S.; Baker, G. A.; Zhao, H. Ether- and alcoholfunctionalized task-specific ionic liquids: attractive properties and applications. Chem. Soc. Rev. 2012, 41, 4030-4066.

(11) Handy, S. T. Greener Solvents. Chem. - Eur. J. 2003, 9, 29382944.

(12) Ionic Liquids; Kirchner, B., Ed.; Springer: Heidelberg, 2009; p 290.

(13) Sasikumar, B.; Arthanareeswaran, G.; Ismail, A. F. Recent progress in ionic liquid membranes for gas separation. J. Mol. Liq. 2018, 266, 330-341.

(14) Rynkowska, E.; Fatyeyeva, K.; Kujawski, W. Application of polymer-based membranes containing ionic liquids in membrane separation processes: a critical review. Rev. Chem. Eng. 2018, 34, 341363.

(15) Le Bideau, J.; Viau, L.; Vioux, A. Ionogels, ionic liquid based hybrid materials. Chem. Soc. Rev. 2011, 40, 907-925.

(16) Göbel, R.; White, R. J.; Titirici, M.-M.; Taubert, A. Carbonbased ionogels: tuning the properties of the ionic liquid via carbonionic liquid interaction. Phys. Chem. Chem. Phys. 2012, 14, 59925997.

(17) Gallastegui, A.; Porcarelli, L.; Palacios, R. E.; Gómez, M. L.; Mecerreyes, D. Catechol-Containing Acrylic Poly(ionic liquid) Hydrogels as Bioinspired Filters for Water Decontamination. ACS Appl. Polym. Mater. 2019, 1, 1887-1895.

(18) Yuan, J.; Mecerreyes, D.; Antonietti, M. Poly(ionic liquid)s. Prog. Polym. Sci. 2013, 38, 1009-1036.

(19) Döbbelin, M.; Azcune, I.; Bedu, M.; Ruiz de Luzuriaga, A.; Genua, A.; Jovanovski, V.; Cabañero, G.; Odriozola, I. Synthesis of Pyrrolidinium-Based Poly(ionic liquid) Electrolytes with Poly(ethylene glycol) Side Chains. Chem. Mater. 2012, 24, 1583-1590.

(20) Eftekhari, A.; Saito, T. Synthesis and properties of polymerized ionic liquids. Eur. Polym. J. 2017, 90, 245-272.
(21) Zimmermann, J.; Ondruschka, B.; Stark, A. Efficient Synthesis of 1,3-Dialkylimidazolium-Based Ionic Liquids. Org. Process Res. Dev. 2010, 14, 1102-1109.

(22) Depuydt, D.; van den Bossche, A.; Dehaen, W.; Binnemans, K. Halogen-free synthesis of symmetrical 1,3-dialkylimidazolium ionic liquids using non-enolisable starting materials. RSC Adv. 2016, 6, 8848-8459.

(23) Tröger-Müller, S.; Brandt, J.; Antonietti, M.; Liedel, C. Green Imidazolium Ionics-From Truly Sustainable Reagents to Highly Functional Ionic Liquids. Chem. - Eur. J. 2017, 23, 11810-11817.

(24) Sirviö, J. A.; Visanko, M.; Liimatainen, H. Synthesis of imidazolium-crosslinked chitosan aerogel and its prospect as a dye removing adsorbent. RSC Adv. 2016, 6, 56544-56548.

(25) Krannig, K.-S.; Esposito, D.; Antonietti, M. Highly Efficient Transfer of Amino Groups to Imidazolium Entities for Polymer Coupling and Cross-Linking. Macromolecules 2014, 47, 2350-2353.

(26) Grygiel, K.; Kirchhecker, S.; Gong, J.; Antonietti, M.; Esposito, D.; Yuan, J. Main-Chain Polyimidazolium Polymers by One-Pot Synthesis and Application as Nitrogen-Doped Carbon Precursors. Macromol. Chem. Phys. 2017, 218, 1600586.

(27) Gong, J.; Lin, H.; Grygiel, K.; Yuan, J. Main-chain poly(ionic liquid)-derived nitrogen-doped micro/mesoporous carbons for $\mathrm{CO} 2$ capture and selective aerobic oxidation of alcohols. Applied Materials Today 2017, 7, 159-168.

(28) Lindner, J.-P. Imidazolium-Based Polymers via the PolyRadziszewski Reaction. Macromolecules 2016, 49, 2046-2053.

(29) Dani, A.; Crocellà, V.; Magistris, C.; Santoro, V.; Yuan, J.; Bordiga, S. Click-based porous cationic polymers for enhanced carbon dioxide capture. J. Mater. Chem. A 2017, 5, 372-383. 